\title{
Rewards can be used effectively with repeated exposure to increase liking of vegetables in 4-6-year-old children
}

\author{
Nadia Corsini ${ }^{1, *}$, Amy Slater ${ }^{2}$, Adam Harrison ${ }^{3}$, Lucy Cooke ${ }^{4}$ and David N Cox ${ }^{1}$ \\ ${ }^{1}$ CSIRO Food and Nutritional Sciences, PO Box 10041, Adelaide BC, South Australia 5000, Australia: ${ }^{2}$ School \\ of Psychology, Flinders University, Adelaide, South Australia, Australia: ${ }^{3}$ Research Centre for Injury Studies, \\ Flinders University, Adelaide, South Australia, Australia: ${ }^{4}$ Department of Epidemiology and Public Health, \\ University College London, London, UK
}

Submitted 1 October 2010: Accepted 7 July 2011: First published online 7 September 2011

\begin{abstract}
Objective: To examine whether parents offering a sticker reward to their child to taste a vegetable the child does not currently consume is associated with improvements in children's liking and consumption of the vegetable.

Design: A randomized controlled trial evaluated the effectiveness of exposure only (EO) and exposure plus reward $(\mathrm{E}+\mathrm{R})$, relative to a control group, on children's liking and consumption of a target vegetable. Assessments were conducted at baseline and 2 weeks from baseline (post-intervention). Follow-up assessments were conducted at 4 weeks and 3 months from baseline.

Setting: The study took place in Adelaide, South Australia. Participants were selfselected in response to local media advertisements seeking to recruit parents finding it difficult to get their children to eat vegetables.

Subjects: Participants were 185 children (110 boys, seventy-five girls) aged $4-6$ years and their primary caregiver/parent (172 mothers, thirteen fathers).

Results: The E $+\mathrm{R}$ group was able to achieve more days of taste exposure. Both EO and $\mathrm{E}+\mathrm{R}$ increased liking at post-intervention compared with control and no further change occurred over the follow-up period. All groups increased their intake of the target vegetable at post-intervention. Target vegetable consumption continued to increase significantly over the follow-up period for $\mathrm{E}+\mathrm{R}$ and control but not for EO. Conclusions: The findings provide support for the effectiveness of using a sticker reward with a repeated exposure strategy. In particular, such rewards can facilitate the actual tastings necessary to change liking.
\end{abstract}

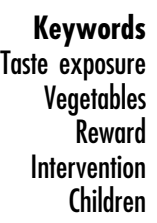

The health benefits of vegetable consumption are widely reported $^{(1)}$. Despite the documented benefits however, children's vegetable consumption in Australia $^{(2)}$ and elsewhere ${ }^{(3)}$ falls well below recommended levels.

There is good evidence that repeated taste exposure to vegetables has a positive influence on children's liking and consumption of vegetables ${ }^{(4,5)}$. Repeated exposure can be facilitated by parents, whose vegetable consumption is possibly the strongest predictor of their children's vegetable consumption ${ }^{(6)}$. Children's liking for vegetables has also been shown to be an important predictor of their consumption ${ }^{(7,8)}$. Thus, encouraging parents to give their children repeated opportunities to taste vegetables is likely to be an important strategy for increasing children's liking for and subsequent consumption of vegetables.

Although repeated exposure can improve liking, getting children to taste vegetables can be challenging. Food neophobia typically peaks between the ages of 2 and 6 years and is characterized by a reluctance to try unfamiliar foods ${ }^{(9)}$, particularly vegetables ${ }^{(6,10,11)}$. Therefore strategies are needed to encourage children to taste foods on enough occasions so that the foods become liked and integrated into the diet. Using rewards as positive reinforcement (termed 'instrumental feeding') is one technique that is widely used by parents to encourage children to eat vegetables ${ }^{(12,13)}$.

The principle of positive reinforcement describes the strengthening of behaviour that occurs as a consequence of receiving a positive outcome. Any reward (stickers, small toys) can be a potential positive reinforcer for behaviour such as tasting a vegetable. Two studies have shown that children's intake or rated liking of vegetables increases when the children are offered a reward ${ }^{(14,15)}$. However, one study has found that reward is no more effective than simply asking the child to taste a small amount the vegetable ${ }^{(14)}$. Indeed, another study suggests that reward may limit the effectiveness of taste exposure $^{(5)}$. Adding further complexity are studies that suggest offering a reward for eating a food may decrease liking 
for that food ${ }^{(16-18)}$, although these studies used foods that are generally accepted (snack foods, milk drinks, juice). Importantly, no study has investigated the effectiveness of rewards with children who have high neophobia and who may be reluctant to taste vegetables at all without some form of encouragement. It follows that the reward will positively reinforce repeated taste exposure, which in turn will facilitate improved liking and consumption of vegetables.

The study by Wardle $e t a l .{ }^{(4)}$ is the only one to examine the effectiveness of repeated taste exposure to a tastedbut-disliked vegetable when led by parents in a home setting. Parents offered a target vegetable every day for $14 \mathrm{~d}$ and children placed stickers on a chart. The study successfully increased both liking and consumption of a target vegetable following a 2-week intervention. Although obtaining stickers was not contingent upon tasting, it is possible that the stickers constituted a reward. If so, the extent to which the sticker reward facilitates repeated tastings is unknown. Lacking a follow-up assessment, it is also unknown whether liking and consumption are maintained once rewards cease. A closer examination of the impact of rewards in the home context is therefore required.

Additionally, repeated exposure typically focuses on a single target vegetable; however, increased liking for one vegetable may positively impact vegetable consumption in general. Measuring children's vegetable intake at baseline and post-intervention would reveal whether effects are seen on everyday consumption of vegetables.

The present study examined whether parents offering a sticker reward to taste a target vegetable is associated with improvements in children's liking and consumption of the target vegetable. The study compared exposure only $(\mathrm{EO})$, exposure plus reward $(\mathrm{E}+\mathrm{R})$ and a control condition. Based on Wardle et $a l^{(4)}$, we expected both $\mathrm{EO}$ and $\mathrm{E}+\mathrm{R}$ to improve liking and consumption relative to control at post-intervention but no hypotheses are made regarding a difference in magnitude of improvement between $\mathrm{EO}$ and $\mathrm{E}+\mathrm{R}$. We expected that $\mathrm{E}+\mathrm{R}$ would positively reinforce children tasting the vegetable, thus improving the number of tastes achieved in the intervention. Follow-up assessments were conducted to determine if positive intervention effects were sustained in the medium term. Furthermore, total dietary vegetable consumption was examined in the medium term to explore if the child's improved liking for a target vegetable was associated with improvements in general vegetable consumption.

\section{Methods}

\section{Design}

A randomized controlled trial evaluated the effectiveness of $\mathrm{EO}$ and $\mathrm{E}+\mathrm{R}$, relative to a control group, on children's liking and consumption of a target vegetable.

\section{Participants}

Participants were self-selected in response to media advertisements seeking to recruit parents in Adelaide, South Australia, finding it difficult to get their children to eat vegetables. Eligibility criteria included the child's age (4-6.99 years), parent commitment to undertake a short activity daily for 2 weeks, willingness to have a fieldworker visit the home on four occasions and ability to communicate in English. Three cohorts of participants were recruited over 9 months. CSIRO Human Research Ethics Committee approved the study. Parents were given a voucher (\$AU 10) to purchase vegetables.

\section{Procedures}

Following eligibility screening, participants were sent information packs and consent forms. Treatment condition was allocated by the trial manager using sequential number allocation as consent forms were processed.

Data collection was carried out by a market research company using fieldworkers experienced in working with children and collecting data in homes. A standard assessment protocol was developed and training provided.

In preparation for the baseline assessment, parents identified six vegetables that they were finding it difficult to get their child to eat. Parents were advised to choose vegetables that would be quick and easy to prepare on a daily basis and avoid vegetables that their child disliked extremely. Parents prepared vegetables as they normally would (e.g. raw or cooked), providing half a cup of each in small pieces. The addition of oil or butter was permitted if this was typical but sauces and herbs/spices were discouraged.

At baseline fieldworkers introduced the procedures to the child as a vegetable tasting game. The procedures described in Wardle et $a l^{(4)}$ were followed to assess liking, assign the target vegetable and assess intake of this target (see paper for details). The target vegetable was the vegetable ranked fourth in a forced-choice elimination activity that assisted the child to rank the six vegetables from most to least liked (most liked $=$ rank 1, least liked $=$ rank 6). Tasted and non-tasted vegetables could be ranked. Parents were present during the assessment but were occupied completing a questionnaire.

\section{Measures}

Target vegetable liking and consumption were assessed at four time points: baseline, 2 weeks from baseline (postintervention), 4 weeks from baseline (follow-up 1) and 3 months from baseline (follow-up 2). Child usual vegetable intake was assessed at baseline, follow-up 1 and follow-up 2.

\section{Target vegetable liking ratings}

Children's rated liking of the target vegetable (together with five other vegetables) was assessed using a 3-point visual 'faces' scale ${ }^{(4,19,20)}$ : 'yummy' (smiling), 'just okay' (neutral face) and 'yucky' (frowning). This replicated the 
procedure described by Wardle et al. ${ }^{(4)}$, including scoring ( 1,0 and -1 , respectively). A score of -2 was given for children who refused to taste the vegetable. The dependent variable is the rating obtained for the target vegetable only.

\section{Target vegetable consumption}

Vegetable consumption was measured by presenting half a cup of one vegetable (the target vegetable) and asking children to eat as much as they liked and to stop when they had eaten enough. Target vegetable consumption was measured to the nearest gram by weighing the cup pre- and post-consumption.

\section{Children's usual vegetable intake}

Children's usual vegetable intake was measured in two ways, based on the parent-report measure, the Children's Dietary Questionnaire ${ }^{(21)}$. To measure variety, parents used a checklist of twenty-three vegetables to indicate how many vegetables child consumed in the past week. To measure frequency of vegetable consumption, parents indicated how often child consumed vegetables on the previous day, ranging from nil to four times (score 0 to 4 ).

\section{Demographics and other measures}

Demographic information was obtained for the parent and child at baseline. Child and parent food neophobia was measured using a ten-item parent-report scale, the Food Neophobia Scale (FNS) ${ }^{(9,22)}$. Scores range from 0 to 70 with higher scores indicating greater neophobia.

\section{Intervention}

Intervention allocation was revealed immediately following baseline assessment. Parents received the intervention materials (or a letter for the control group) and were told to commence the intervention on the following day using the target vegetable. Children were assessed by the same fieldworker throughout the study.

\section{Exposure only}

Consistent with Wardle et $a l .{ }^{(4)}$, the EO intervention required the parent to present and ask their child to taste a small piece of the target vegetable every day for 2 weeks. Parents were asked to keep the preparation method constant and a lowkey approach was emphasized. Parents were also advised to present the vegetable at a predictable time, before a meal or snack, when the child would be hungry.

Standardized instructions were provided in a booklet which included a flowchart with suggestions about how to respond to a child who refused to taste. Specifically, parents were asked to model tasting the vegetable (refusal 1) and cut the vegetable into smaller pieces (refusal 2) and stop after that.

\section{Exposure plus rewards}

The $\mathrm{E}+\mathrm{R}$ group followed the $\mathrm{EO}$ procedures with the addition of a 14-d chart, replicating the procedure in
Wardle et $a l^{(4)}$ for the 'Exposure group'. Stickers with 'yummy', 'just okay' and 'yucky' faces were provided as a reward for tasting the vegetable. Parents were told to provide mild encouragement (i.e. 'you can use your sticker to tell me how the vegetable tastes') rather than controlling statements (i.e. 'if you don't taste it you can't have a sticker').

\section{Control}

The control group did not receive the standardized instruction booklet. Parents were asked to maintain their normal feeding behaviours for the course of the study. The target vegetable was not revealed to this group, although parents were asked to prepare half a cup of a given (target) vegetable for post-intervention and followup assessments. At the end of the follow-up 2 assessment, parents were fully briefed about the intervention and given the instruction booklet.

Forty parents (fourteen from $\mathrm{E}+\mathrm{R}$, sixteen from $\mathrm{EO}$ and ten controls) were interviewed post-intervention to elicit their behaviour during the trial. A detailed discussion of the findings is beyond the scope of the present paper but is briefly reported.

\section{Number of tastes achieved during intervention}

Parents used a diary to record the outcome of each exposure trial (i.e. child tasted or refused vegetable). Putting the vegetable into the mouth and then spitting it out counted as a taste. It was hoped that at least ten successful taste exposures would be achieved, as this number has been associated with improvements in liking ${ }^{(4)}$.

\section{Statistical analysis}

An independent-samples $t$ test was used to determine if $\mathrm{E}+\mathrm{R}(n 57)$ and $\mathrm{EO}(n 58)$ differed on number of tastes achieved during the intervention.

To determine impact of treatment condition on target vegetable liking ratings, a repeated-measures linear mixed-effects model was run with two factors: treatment condition (EO, E+R, control) as the between-subjects factor and time (baseline, post-intervention) as the within-subjects factor. For target vegetable consumption, a negative binomial generalized linear model was run to accommodate a positively skewed distribution containing a number of ' 0 ' intake scores. If a significant interaction was found between time and treatment condition, post hoc comparisons were conducted to determine for which treatment conditions significant changes occurred.

Participants were excluded from the analyses if the target vegetable was tasted fewer than nine times during the 2-week exposure period, resulting in twenty-seven cases from $\mathrm{EO}$ and fourteen cases from $\mathrm{E}+\mathrm{R}$ being excluded. The final sample was: control ( $n$ 64), EO ( $n$ 35) and $\mathrm{E}+\mathrm{R}(n$ 45). The desired sample size was fifty-two per group (based on a power of 0.8 and effect size of $0 \cdot 25)$; thus the analyses were somewhat underpowered. 
The models described above were also run to examine changes in target vegetable liking ratings and target vegetable consumption over the follow-up period. Additionally, repeated-measures linear mixed-effects models were run to examine changes in children's usual vegetable intake, specifically (i) variety of vegetables consumed and (ii) frequency of vegetable consumption.

\section{Data screening and preliminary analyses}

Multivariate outliers were found to be within an acceptable range ( $Z$-scores of the residuals between $\pm 3 \cdot 29$ ).

Child's age was included as a covariate in all analyses as ANOVA indicated that the three groups differed $(F(2,134)=3 \cdot 68, P<0 \cdot 05)$. No group differences were found for child's gender, food neophobia score and usual vegetable intake at baseline.

\section{Results}

\section{Sample characteristics}

One hundred and eighty-eight participants were randomized to control, EO or E+R (Fig. 1). Participants who completed baseline testing were 185 children (110 boys, seventy-five girls) and their primary caregiver (172 mothers, thirteen fathers). Sample characteristics are shown in Table 1. Unsurprisingly, child food neophobia scores were high, showing a general unwillingness to try novel foods. Neophobia was higher than a recent study of 371 Australian children aged 2 to 5 years that reported average scores of $36 \cdot 59(\mathrm{SD} 14 \cdot 05)^{(11)}$.

\section{Intervention target vegetables}

Target vegetables included twenty-two different vegetables, the most common being carrot ( $n$ 20), cauliflower ( $n$ 17), cucumber $(n 17)$ and broccoli ( $n$ 16).

\section{Number of tastes achieved during intervention}

Parent diaries were returned by 115 participants (94\%) in $\mathrm{EO}$ and $\mathrm{E}+\mathrm{R}$ who commenced the intervention. The majority of parents ( $86 \%$ ) offered the target vegetable on a least ten occasions over the 2-week exposure period; however, just over half of the children (56\%) achieved ten taste exposures. Seventy per cent of the children tasted their target vegetable at least nine times, hence this was chosen as the analysis sample. Examination of the parent diaries indicated failure was due mainly to the child refusing to taste rather than the parent failing to offer the vegetable.

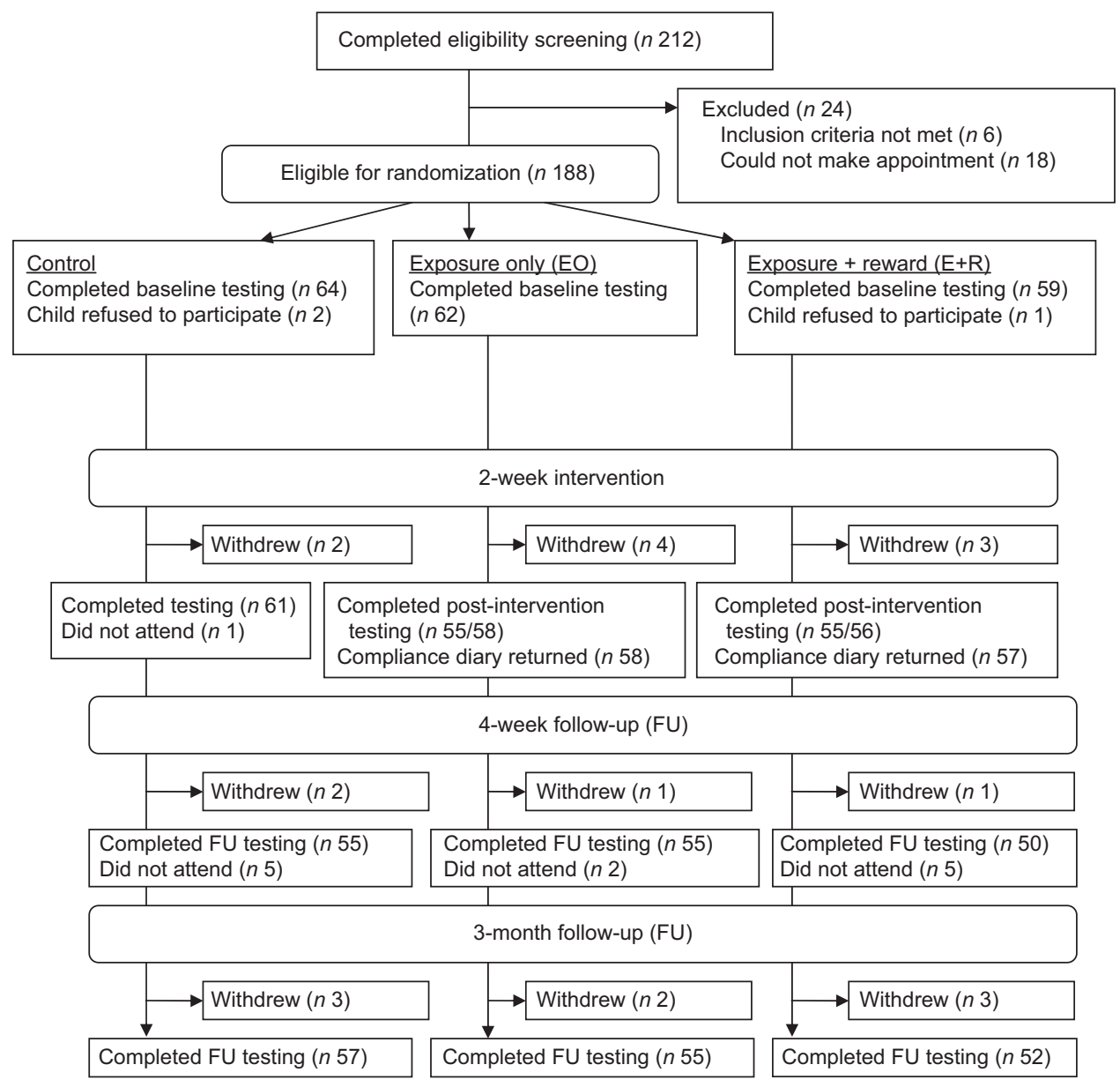

Fig. 1 Diagram showing the flow of participants through each stage of the randomized controlled trial 
Table 1 Sample characteristics for children and parents: randomized controlled trial conducted among children aged 4-6 years and their primary caregiver/parent, Adelaide, South Australia

\begin{tabular}{|c|c|c|c|c|c|}
\hline $\begin{array}{l}\text { Child characteristics } \\
(n \text { 185) }\end{array}$ & $n$ & $\%$ & $\begin{array}{l}\text { Parent characteristics } \\
(n \text { 180) }\end{array}$ & $n$ & $\%$ \\
\hline Gender & & & Gender & & \\
\hline Male & 110 & 60 & Male & 13 & 7 \\
\hline Female & 75 & 40 & Female & 172 & 93 \\
\hline Position in family & & & Education & & \\
\hline Oldest & 77 & 42 & High school or less & 31 & 17 \\
\hline Middle & 23 & 12 & Technical certificate & 36 & 20 \\
\hline Youngest & 43 & 23 & University & 113 & 61 \\
\hline Siblings & & & & Mean & SD \\
\hline Only child & 36 & 20 & Age (years) & $\overline{39 \cdot 15}$ & $\overline{4.91}$ \\
\hline 1 sibling & 89 & 48 & Food neophobiat & $23 \cdot 44$ & $10 \cdot 11$ \\
\hline \multirow[t]{2}{*}{$2+$ siblings } & 55 & 30 & & & \\
\hline & Mean & SD & & & \\
\hline Age (years) & $\overline{5 \cdot 16}$ & 0.84 & & & \\
\hline Food neophobiat & $51 \cdot 82$ & $13 \cdot 49$ & & & \\
\hline
\end{tabular}

Note: demographic information was missing for five parents.

tScores range from 0 to 70 with higher scores indicating greater neophobia.

Table 2 Children's baseline target vegetable liking ratings by study group: randomized controlled trial conducted among children aged 4-6 years and their primary caregiver/parent, Adelaide, South Australia

\begin{tabular}{|c|c|c|c|c|c|c|}
\hline \multirow[b]{2}{*}{ Liking rating } & \multicolumn{2}{|c|}{$\begin{array}{c}\text { Control } \\
(n \text { 61) }\end{array}$} & \multicolumn{2}{|c|}{$\begin{array}{c}\text { EO } \\
(n 34)\end{array}$} & \multicolumn{2}{|c|}{$\begin{array}{l}\mathrm{E}+\mathrm{R} \\
(n+43)\end{array}$} \\
\hline & $n$ & $\%$ & $n$ & $\%$ & $n$ & $\%$ \\
\hline Yummy & 16 & 25 & 7 & 20 & 9 & 20 \\
\hline Neutral & 20 & 31 & 17 & 49 & 18 & 40 \\
\hline Yucky & 23 & 36 & 10 & 29 & 17 & 38 \\
\hline Refused to taste & 5 & 8 & 1 & 3 & 1 & 2 \\
\hline
\end{tabular}

$E O$, exposure only group; $E+R$, exposure plus reward group.

Note: sample of participants who achieved nine or more taste exposures.

Independent-samples $t$ tests were conducted to compare neophobia scores, baseline target vegetable liking scores and baseline target vegetable consumption between children with nine or more $v$. fewer than nine tastes. No significant differences were found on neophobia scores $(t(109)=0 \cdot 70, P=0 \cdot 49)$ or on baseline target vegetable consumption $(t(113)=0.09, P=0.93)$. Baseline target vegetable liking was significantly lower in children with fewer than nine tastes (mean -0.63 , SD 0.97 ) than in children with nine or more tastes (mean $-0 \cdot 19$, SD $0 \cdot 78 ; t(113)=-2 \cdot 58, P=0 \cdot 01)$.

\section{Baseline liking for target vegetable}

Despite parents selecting vegetables that their child disliked, some of the vegetables were reported as liked at baseline (see Table 2). Of the analysis sample, $20 \cdot 3 \%$ of children started the study with a target vegetable that was rated 'yummy'. Of the children who made yummy ratings at baseline, $71 \cdot 4 \%$ indicated 'yummy' at post-intervention.

Unsurprisingly, children who refused to taste the target vegetable at the initial baseline tasting had significantly

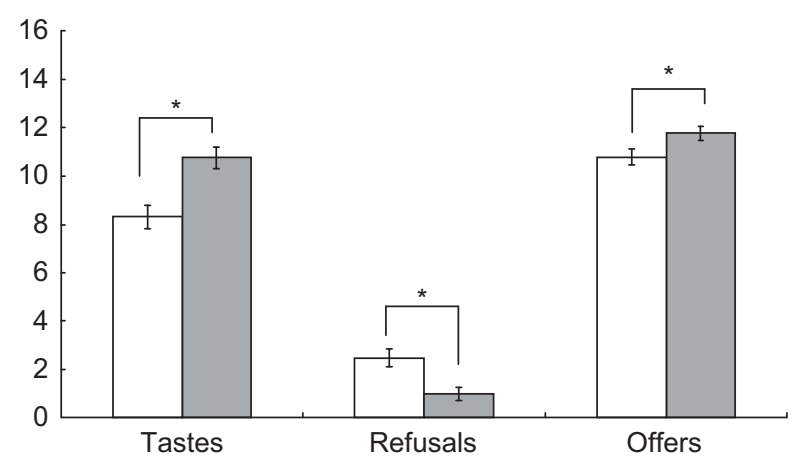

Fig. 2 Number of days of taste exposure (tastes), refusals to taste (refusals) and offers of the vegetable by the parent (offers), for the EO (exposure only, $n 58 ; \square$ ) and the E+R (exposure plus reward, $n 57 ; \square$ ) groups, in a randomized controlled trial conducted among children aged 4-6 years and their primary caregiver/parent, Adelaide, South Australia. Values are means with their standard deviations represented by vertical bars. Mean values were significantly different between groups: ${ }^{\star} P<0.05$

higher neophobia scores (mean 58.93, SD 11.90) than children who tasted their vegetable at baseline (mean $51 \cdot 18$, SD $13 \cdot 47 ; t(178)=2 \cdot 15, P=0 \cdot 033)$.

\section{Effect of exposure only and exposure plus reward on number of tastes achieved}

$\mathrm{E}+\mathrm{R}$ achieved more days of taste exposure $(t(113)=$ $-3 \cdot 78, \quad P<0 \cdot 05)$ and fewer refusals $(t(113)=3 \cdot 18$, $P<0 \cdot 05$ ) compared with EO (see Fig. 2). Additional analysis showed $\mathrm{E}+\mathrm{R}$ also offered the vegetable more often than EO $(t(113)=-2 \cdot 22, P<0 \cdot 05)$. Furthermore, $77 \cdot 2 \%$ of children in $\mathrm{E}+\mathrm{R}$ achieved ten or more tastes, compared with only $37.95 \%$ of children in EO. For nine or more tastes, the frequencies were $78.9 \%$ and $60 \cdot 3 \%$, respectively. 


\section{Effect on target vegetable liking ratings}

From baseline to post-intervention there was a significant group-by-time interaction $(F(1,134)=3 \cdot 62, P=0 \cdot 029)$, which indicated that liking increased significantly for both EO (change $0 \cdot 48$, sE $0 \cdot 15, P=0 \cdot 002$ ) and $\mathrm{E}+\mathrm{R}$ (change 0.58, se $0 \cdot 13, P<0 \cdot 001$ ) but not for control (Fig. 3). Post hoc analyses revealed that liking ratings for $\mathrm{EO}$ and $\mathrm{E}+\mathrm{R}$ were both significantly higher than for control $(P=0 \cdot 04$ and $0 \cdot 02$, respectively) but not significantly different from each other at post-intervention $(P=1 \cdot 00)$.
There was no overall change in liking from postintervention to 4-week follow-up and 3 month follow-up $(F(2,128)=0 \cdot 45, P>0 \cdot 63$; Fig. 4). At 3-month follow-up, $58 \%$ of children in $\mathrm{E}+\mathrm{R}$ and $53 \%$ of children in EO rated their target vegetable as 'yummy', compared with $38 \%$ of the control group children.

\section{Effect on target vegetable consumption}

Contrary to prediction, there was a significant effect of time only $\left(\chi^{2}(1)=22 \cdot 98, P<0 \cdot 001\right)$, indicating that all

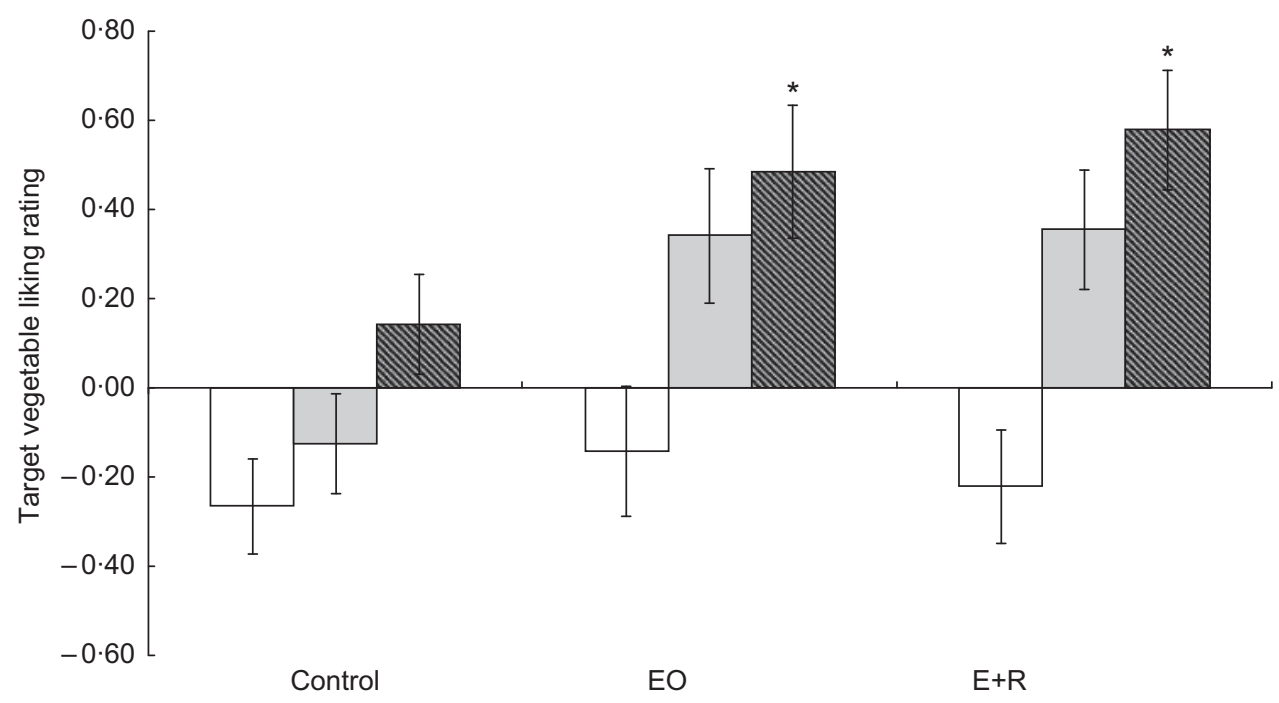

Fig. 3 Target vegetable liking ratings for the target vegetable at baseline $(\square)$ and post-intervention $(\square)$, and magnitude of change in rating from baseline to post-intervention ( $\mathbb{N}$ ), for the control (baseline, $n$ 64; post-intervention, $n$ 61), EO (exposure only; baseline, $n$ 35; post-intervention, $n 34$ ) and $\mathrm{E}+\mathrm{R}$ (exposure plus reward; baseline, $n$ 45; post-intervention, $n$ 43) groups, in a randomized controlled trial conducted among children aged 4-6 years and their primary caregiver/parent, Adelaide, South Australia. Hedonics ratings represent 'yummy' above the '0' point and 'yucky' below it. Values are means with their standard errors represented by vertical bars. Change between baseline and post-intervention was significant: ${ }^{\star} P<0.05$

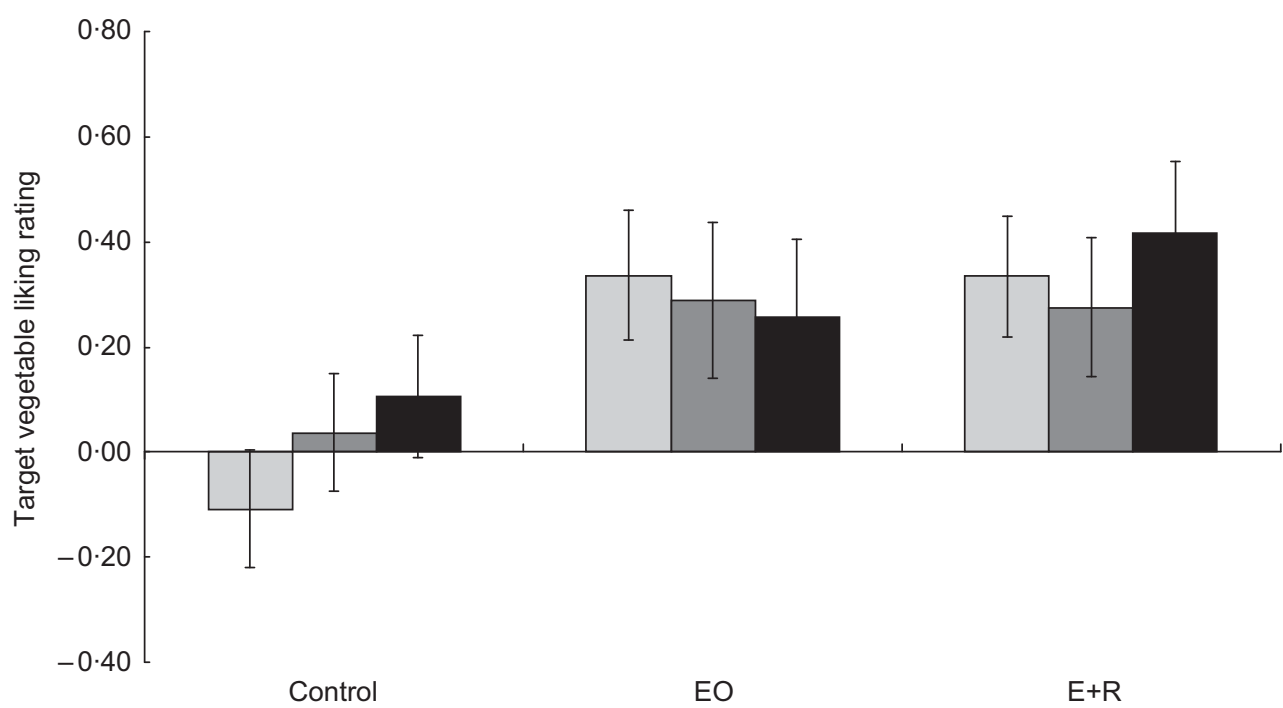

Fig. 4 Target vegetable liking ratings for the target vegetable at post-intervention ( $\square$ ), 4-week follow-up ( $\square$ ) and 3-month follow-up

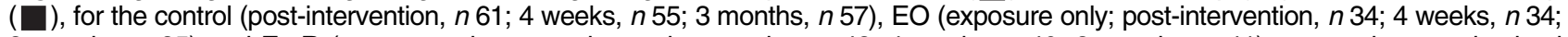
3 months, $n$ 35) and $\mathrm{E}+\mathrm{R}$ (exposure plus reward; post-intervention, $n$ 43; 4 weeks, $n$ 40; 3 months, $n$ 41) groups, in a randomized controlled trial conducted among children aged 4-6 years and their primary caregiver/parent, Adelaide, South Australia. Hedonics ratings represent 'yummy' above the '0' point and 'yucky' below it. Values are means with their standard errors represented by vertical bars 


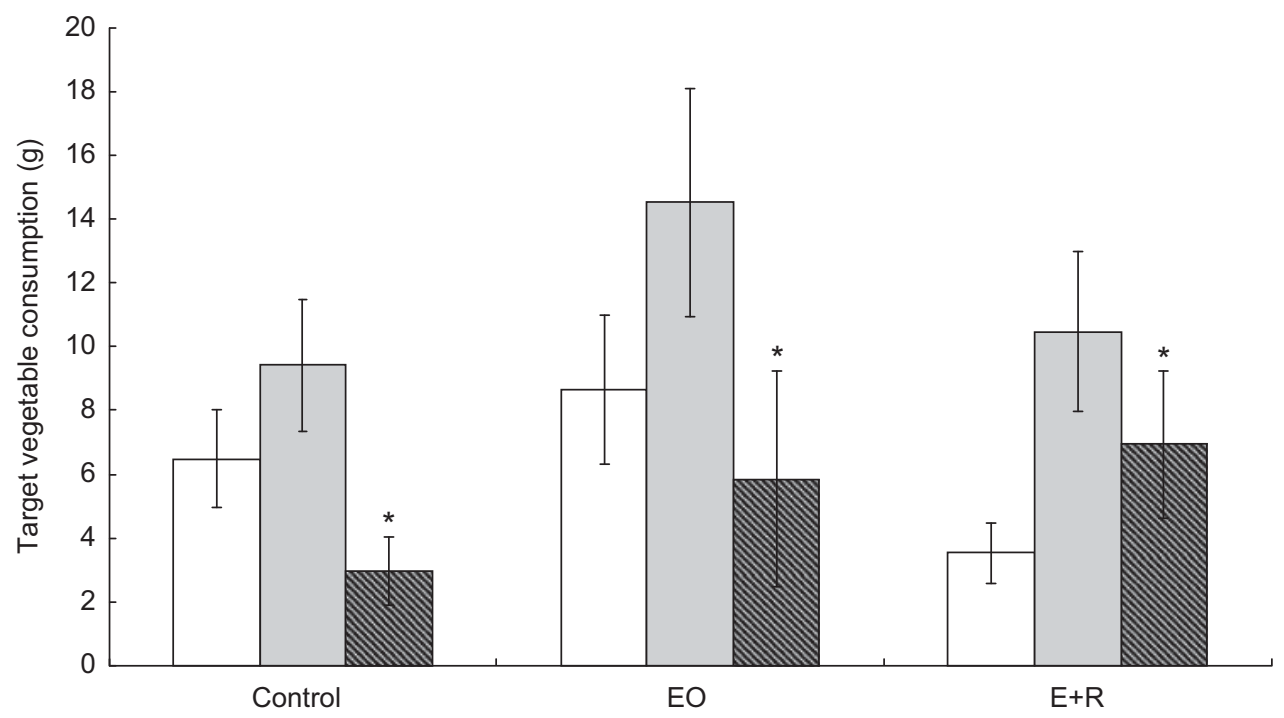

Fig. 5 Target vegetable consumption on the previous day $(\mathrm{g})$ at baseline $(\square)$ and post-intervention ( $\square$ ), and magnitude of change in consumption from baseline to post-intervention ( $\mathbb{N}$ ), for the control (baseline, $n$ 64; post-intervention, $n 61$ ), EO (exposure only; baseline, $n$ 35; post-intervention, $n 34$ ) and $\mathrm{E}+\mathrm{R}$ (exposure plus reward; baseline, $n 45$; post-intervention, $n$ 43) groups, in a randomized controlled trial conducted among children aged 4-6 years and their primary caregiver/parent, Adelaide, South Australia. Values are means with their standard errors represented by vertical bars. Change between baseline and post-intervention was significant: ${ }^{*} P<0.05$

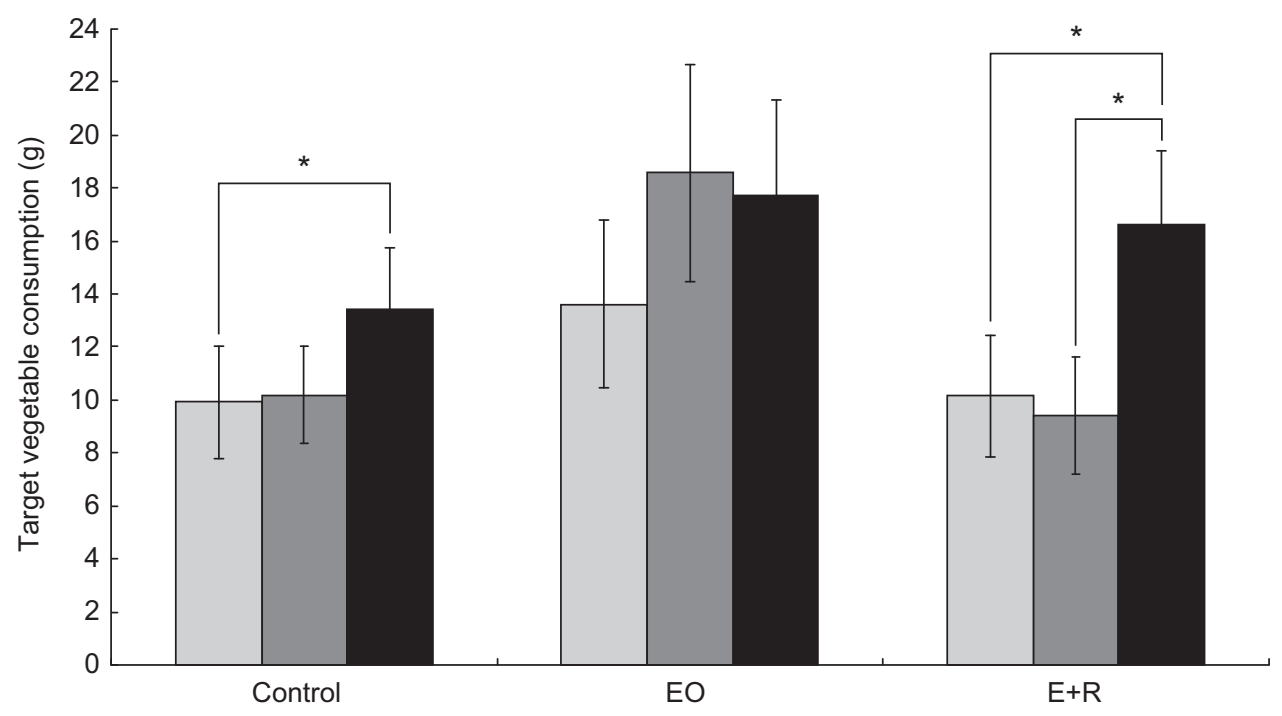

Fig. 6 Target vegetable consumption on the previous day $(\mathrm{g})$ at post-intervention ( $\square$ ), 4-week follow-up ( $\square$ ) and 3-month follow-up ( $)$, for the control (post-intervention, $n 61 ; 4$ weeks, $n 55$; 3 months, $n 57$ ), EO (exposure only; post-intervention, $n 34$; 4 weeks, $n 34$; 3 months, $n$ 35) and $\mathrm{E}+\mathrm{R}$ (exposure plus reward; post-intervention, $n 43$; 4 weeks, $n$ 40; 3 months, $n$ 41) groups, in a randomized controlled trial conducted among children aged 4-6 years and their primary caregiver/parent, Adelaide, South Australia. Values are means with their standard errors represented by vertical bars. Mean values were significantly different between time periods: ${ }^{\star} P<0 \cdot 05$

groups increased their intake of the target vegetable from baseline (Fig. 5).

There was a significant group-by-time interaction $\left(\chi^{2}(4)=14 \cdot 88, \quad P=0 \cdot 005\right)$ over the follow-up period (Fig. 6). For $\mathrm{E}+\mathrm{R}$, target vegetable consumption increased significantly from post-intervention to 3 months (change 6.47, SE $2 \cdot 26, P=0 \cdot 013$ ) and increased significantly from 4 weeks to 3 months (change $7 \cdot 23$, sE $1 \cdot 49, P<0 \cdot 001$ ). For the control group consumption increased significantly between post-intervention and 3-month follow-up (change $3 \cdot 51$, se $1 \cdot 22, P=0 \cdot 012$ ).

\section{Effect on child's usual vegetable intake}

For variety of vegetable consumption there was a significant effect of time only $(F(2,126)=30 \cdot 23, P<0 \cdot 001)$, indicating an overall increase in the variety of vegetables consumed. For frequency of vegetable consumption there was a significant group-by-time interaction $(F(4,129)=2 \cdot 81, \quad P<0 \cdot 05)$. 


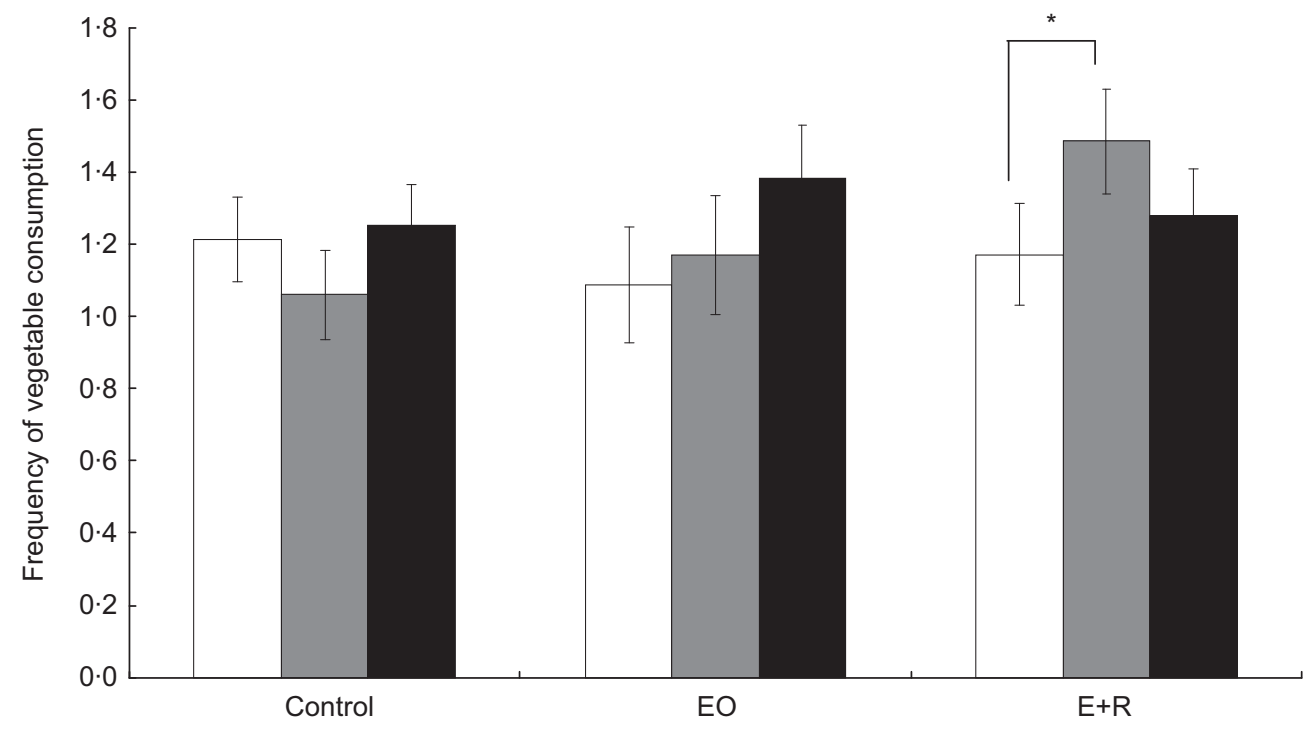

Fig. 7 Frequency of vegetable consumption on the previous day at baseline ( $\square$ ), 4-week follow-up ( $\square$ ) and 3-month follow-up ( $\square$ ), for the control (baseline, $n 63,4$ weeks, $n 56$; 3 months, $n 57$ ), EO (exposure only; baseline, $n 34,4$ weeks, $n 32 ; 3$ months, $n 34$ ) and $\mathrm{E}+\mathrm{R}$ (exposure plus reward; baseline, $n 44$, 4 weeks, $n 40 ; 3$ months, $n 41$ ) groups, in a randomized controlled trial conducted among children aged 4-6 years and their primary caregiver/parent, Adelaide, South Australia. Values are means with their standard errors represented by vertical bars. Mean values were significantly different between time periods: ${ }^{*} P<0.05$

For $\mathrm{E}+\mathrm{R}$, frequency of vegetable consumption increased from baseline to 4 weeks (change $0 \cdot 32$, sE $0 \cdot 12, P<0 \cdot 05$; see Fig. 7).

\section{Control group parent interviews}

Nine of the ten control participants sampled reported no change in the way they offered or presented vegetables to their children through involvement in the study. Five participants reported that their child was more willing to eat vegetables.

\section{Discussion}

A randomized controlled trial evaluated the effectiveness of two home-based and parent-led interventions based on taste exposure, $\mathrm{EO}$ and $\mathrm{E}+\mathrm{R}$, relative to a control condition, on 4-6-year-old children's liking and consumption of a target vegetable. This is the first study to compare taste exposure alone and taste exposure combined with rewards in this context. Novel aspects include the addition of a 3-month follow-up period and the assessment of children's everyday vegetable intake.

Consistent with the findings of Wardle et $a l^{(4)}$ and the taste exposure mechanism, both $\mathrm{EO}$ and $\mathrm{E}+\mathrm{R}$ increased liking at post-intervention. The magnitude of improvement in the current study is smaller than that obtained by Wardle and colleagues (approximately 0.5 point $v .1$ point on the liking rating scale). This difference could be due to the high levels of child food neophobia in the current sample. Wardle et al. ${ }^{(4)}$ did not assess child food neophobia and participants were recruited to 'modify their children's acceptance of vegetables', whereas in the present study parents were specifically recruited on the basis that they were finding it difficult to get their children to eat vegetables.

In contrast to Wardle et al.'s study, there was no intervention effect on consumption of the target vegetable, despite the increase in liking scores. However, target vegetable consumption increased from baseline to post-intervention for all conditions, possibly due to overall study involvement or child maturation. Liking may not necessarily lead to a parallel increase in consumption as vegetable intake is likely to be affected by other factors (e.g. variation in hunger and presentation) that it was not possible to control using this parent-led paradigm. However, an attempt was made to minimize the impact of these factors by scheduling the assessments at the same time of day and asking parents to prepare the vegetable in the same way and in the same quantity. However it is unknown whether parents always complied with these instructions.

Demand characteristics might explain the increase in target vegetable consumption observed in the control condition. It is possible that testing procedures and parent preparation of vegetables lead to 'lay' exposure practices, despite the post-intervention interviews suggesting that control parents did not change their feeding practices. There may have been demand effects in the taste test (wanting to please the fieldworker) affecting all children (including controls). The number of children rating their target vegetable as 'yummy' at baseline supports the presence of demand effects.

The non-blinding of participants to the intervention may have introduced some performance bias for participants 
and assessment bias for fieldworkers. Although nonblinding is a potential impact on parent-report measures, it is less likely to influence the ratings provided by the children. With respect to assessment bias, fieldworkers were not part of the study team, visits were conducted independently and there was a standardized protocol which emphasized neutrality.

The addition of reward did not limit the effectiveness of the exposure technique as reported by Wardle et $a l^{(5)}$. $\mathrm{E}+\mathrm{R}$ and $\mathrm{EO}$ increased liking at post-intervention, and there was no evidence that $\mathrm{E}+\mathrm{R}$ improved to a lesser degree. Importantly, liking was maintained in the 3 months following the intervention, after the reward had ceased. Furthermore, $\mathrm{E}+\mathrm{R}$ continued to increase their intake of the target vegetable from baseline to 3-month follow-up and the frequency of usual vegetable consumption at 4 weeks, whereas no such improvements were seen in the EO group. Children in the EO group may have perceived they were being pressured to eat their vegetables. The pressure could have made the vegetable more aversive and account for why the EO group had no improvement in vegetable consumption compared with the control group (which did not pressure children to eat) and the $\mathrm{E}+\mathrm{R}$ group (which offered a small reward).

A practical implication of the findings is that rewards can be used to encourage vegetable consumption in children who are reluctant to taste vegetables. The beneficial impact of reward is consistent with a meta-analysis that concluded that rewards are likely to have a positive impact on behaviour particularly when the initial task (as in the current study) is low in enjoyment and when there is a clear link between the reward and the target behaviour ${ }^{(23)}$.

Parents of highly neophobic children are most in need of strategies to encourage vegetable consumption. Interestingly, although food neophobia was associated with initial refusal to taste, it was not associated with parents' ability to offer vegetables. This implies that parents of neophobic children can successfully carry out a taste exposure intervention. A point of difference from previous taste exposure studies ${ }^{(4,5)}$ is the increased ecological validity associated with the choice of vegetable and its preparation, the responsibility of the parent. As a result the study demonstrates that the technique can be effective across a wide range of vegetables and preparation styles, and, as these were presumably reflective of the household's typical eating habits, can subsequently be incorporated into the child's diet with minimal inconvenience for parents.

Given the difficulty many parents face in getting their children to taste vegetables, knowing that rewards can be used effectively has practical application. Most children (70\%) achieved at least nine taste exposures in 2 weeks. Post-intervention interviews indicated that the intervention was well received by parents who developed skills in persistence in offering vegetables. Repeated exposure is different from the strategies parents typically try (e.g. making vegetables 'fun and exciting', hiding vegetables in other dishes, or forcing the child to eat 'what's good for them'), all of which are less effective than tasting for creating lasting preferences. The exposure strategy also emphasizes an 'eat every day' message in contrast to the 'eat quantity' messages in the current Australian social marketing 'Go for $2 \& 5^{\circledR}$, campaign. Furthermore, the nature of the reward used differed from rewards that parents might typically use to encourage vegetable consumption, such as offering more attractive foods.

Repeated exposure may represent too great a burden for the most hard-pressed parents and greater flexibility may be required. For example, it is possible that some neophobic children may need more exposures to overcome their initial dislike for vegetables and having a longer period in which to achieve the taste exposures, with less pressure to present the vegetable every day, may be helpful. This might also reduce the likelihood of boredom or monotony, often encountered with repeated exposure of single foods ${ }^{(24)}$.

The present study is the second to provide evidence for the effectiveness of repeated taste exposure in a homebased setting. Future work could consider disseminating the exposure intervention to parents by presenting it as an Internet-based programme. It would also be valuable to examine programme effects on psychological constructs (e.g. parent self-efficacy) that are associated with vegetable intake in children.

\section{Conclusions}

The present findings provide support for using rewards together with repeated taste exposure and in particular suggest that rewards can facilitate the actual tastings which are necessary to change perceptions of vegetable liking, and which are especially difficult to achieve in highly picky or neophobic children. This simple taste exposure activity with stickers can be disseminated in a variety of settings to educate parents and caregivers about the importance of repeated exposure.

\section{Acknowledgements}

The project was funded by Horticulture Australia Limited using the vegetable levy and matched funding from the Australian Government. There are no conflicts of interest to report. N.C., A.S., A.H. and D.N.C. conceived of and designed the study. N.C. carried out the data analysis. All authors contributed to the interpretation of results and to the development of the manuscript. The authors thank Ms Kylie Lange for providing statistical advice and assistance.

\section{References}

1. Van Duyn MA \& Pivonka E (2000) Overview of the health benefits of fruit and vegetable consumption for the dietetics professional: selected literature. J Am Diet Assoc 100, 1511-1521. 
2. Magarey A, McKean S \& Daniels L (2006) Evaluation of fruit and vegetable intakes of Australian adults: the National Nutrition Survey 1995. Aust N Z J Public Health 30, 32-37.

3. Yngve A, Wolf A, Poortvliet E et al. (2005) Fruit and vegetable intake in a sample of 11-year-old children in 9 European countries: The Pro Children Cross-sectional Survey. Ann Nutr Metab 49, 236-245.

4. Wardle J, Cooke L, Gibson EL et al. (2003) Increasing children's acceptance of vegetables; a randomized trial of parent-led exposure. Appetite 40, 155-162.

5. Wardle J, Herrera ML, Cooke L et al. (2003) Modifying children's food preferences: the effects of exposure and reward on acceptance of an unfamiliar vegetable. Eur J Clin Nutr 57, 341-348.

6. Cooke L, Wardle J, Gibson EL et al. (2003) Demographic, familial and trait predictors of fruit and vegetable consumption by preschool children. Public Health Nutr 7, 295-302.

7. Gibson EL, Wardle J \& Watts CJ (1998) Fruit and vegetable consumption, nutritional knowledge and beliefs in mothers and children. Appetite 31, 205-228.

8. Resnicow K, Davis-Hearn M, Smith M et al. (1997) Socialcognitive predictors of fruit and vegetable intake in children. Health Psychol 16, 272-276.

9. Pliner P (1994) Development of measures of food neophobia in children. Appetite 23, 147-163.

10. Cooke L, Carnell S \& Wardle J (2006) Food neophobia and mealtime food consumption in 4-5 year old children. Int J Behav Nutr Phys Act 3, 14.

11. Russell CG \& Worsley A (2008) A population-based study of preschoolers' food neophobia and its associations with food preferences. J Nutr Educ Behav 40, 11-19.

12. Campbell KJ, Crawford DA \& Hesketh KD (2007) Australian parents' views on their 5-6-year-old children's food choices. Health Promot Int 22, 11-18.
13. Casey R \& Rozin P (1989) Changing children's food preferences: parent opinions. Appetite 12, 171-182.

14. Hendy HM (1999) Comparison of five teacher actions to encourage children's new food acceptance. Ann Behav Med 21, 20-26.

15. Hendy HM, Williams KE \& Camise TS (2005) 'Kids Choice' school lunch program increases children's fruit and vegetable acceptance. Appetite 45, 250-263.

16. Birch L, Birch D, Marlin DW et al. (1982) Effects of instrumental consumption on children's food preference. Appetite 3, 125-134.

17. Birch LL, Marlin DW \& Rotter J (1984) Eating as the 'means' activity in a contingency: effects on young children's food preference. Child Dev 55, 431-439.

18. Newman J \& Taylor A (1992) Effect of a means-end contingency on young children's food preferences. J Exp Child Psychol 53, 200-216.

19. Birch LL \& Sullivan SA (1991) Measuring children's food preferences. J Sch Health 61, 212-214.

20. Guthrie CA, Rapoport L \& Wardle J (2000) Young children's food preferences: a comparison of three modalities of food stimuli. Appetite 35, 73-77.

21. Magarey A, Golley R, Spurrier N et al. (2009) Reliability and validity of the Children's Dietary Questionnaire; a new tool to measure children's dietary patterns. Int J Pediatr Obes $\mathbf{4}$, 257-265.

22. Pliner P \& Hobden K (1992) Development of a scale to measure the trait of food neophobia in humans. Appetite 19, 105-120.

23. Cameron J, Banko KM \& Pierce WD (2001) Pervasive negative effects of rewards on intrinsic motivation: the myth continues. Behav Anal 24, 1-44.

24. Siegal PS \& Pilgrim FJ (1958) A field study of food monotony. Am J Psychol 71, 756-759. 